\title{
Molecular Docking studies and in-silico ADMET Screening of Some novel Oxazine substituted 9-Anilinoacridines as Topoisomerase II Inhibitors
}

\author{
Rajagopal Kalirajan*, Sundaram Sankar, Selvaraj Jubie, Byran Gowramma \\ Department of Pharmaceutical Chemistry, JSS College of Pharmacy, Constituent college, JSS University, Mysore INDIA.
}

\begin{abstract}
Introduction: In general, 9-aminoacridine derivatives are inhibiting DNA topoisomerase II (topoll) because of their strong activity due to the ability of acridine nucleus to intercalate into DNA base pair. To get insight of the intermolecular interactions, the molecular docking studies are performed at active site of topoisomerase II. Aim: In this study, an attempt is made for identification of potential ligands from oxazine substituted 9-anilino acridines targeted against topoisomerase-II (1ZXM) using molecular modelling and docking studies by using Schrodinger suit-2012 Maestro 9.3 version. Insilco ADMET screening also performed by qikprop module of Schrodinger suit. Results: The relative binding affinity of the designed compounds towards topoisomerase-II (1ZXM) was selected on the basis of docking score, GLIDE score and interaction patterns. Several compounds showed strong hydrogen bonding interactions with amino acid residues and their hydrophobic interactions and other parameters could also explain their potency to inhibit topoisomerase-II (1ZXM). The oxazine substituted 9 -anilino acridine derivatives $1 \mathbf{a}-\mathbf{1 k}$ have good binding affinity with Glide score in the range of -5.7 to -8.06 when compared with the standard ledacrine (-5.24). The ADMET screening of the designed compounds have almost all the properties of the compounds are within the recommended values. Conclusion: Hence, this study provides evidence for consideration of valuable ligands in oxazine substituted 9-anilino acridine derivatives as potential topoisomerase-II inhibitor and further in vitro and in vivo investigations may prove its therapeutic potential
\end{abstract}

Key words: Topoisomerase-II, Acridine, Oxazine, Antitumour, Docking studies, Insilico ADMET screening.

\section{INTRODUCTION}

In general, 9-aminoacridine derivatives work inhibiting DNA topoisomerase II (topoII). Their strong activity was due to the ability of acridine nucleus to intercalate into DNA base pair, stabilizing the DNA-topoII cleavable complex, and forming the so-called 'ternary complex' which involve DNA, intercalated compound and topoII. The poisoning of topoII activity inhibits the religation process and causes lethal double-strand breaks in DNA, leading to cell cycle arrest and apoptosis. The intercalative property was referred to the planar aromatic system of the acridine moiety.
In the same context, acridines have gained strong ground for various biological activities like antimicrobial, ${ }^{1}$ antioxidant, ${ }^{2}$ anticancer, ${ }^{3-5}$ antimalarial, ${ }^{6}$ analgesic, ${ }^{7}$ antileishmanial, ${ }^{8}$ antinociceptive, ${ }^{9}$ acetyl cholinesterase inhibitors ${ }^{10}$ and antiherpes ${ }^{11}$ etc. Amsacrine is the best known compound of 9-anilinoacridines series. It was one of the first DNA-intercalating agents to be considered as a Topoisomerase II inhibitor. The intercalation process is the strongest type of reversible binding to the double helical DNA in compounds with sufficiently large coplanar aromatic chromophore. Moreover, the cytotoxicity of most of the
Submission Date: 08-08-2016; Revision Date: 26-09-2016; Accepted Date: 28-09-2016

DOI: 10.5530/ijper.51.1.15 Correspondence: Dr. R.Kalirajan, Asst.Professor, Dept. of Pharmaceutical Chemistry, J.S.S College of Pharmacy, Ooty-643 001, Tamil Nadu INDIA.

Tel- +91-423-2443393

(Ext-223)

Fax- +91-4232442937

E-mail: rkalirajan@ymail.com rkalirajan@jsscpooty.org.

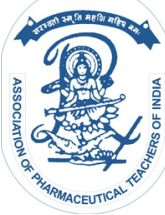

www.ijper.org 
clinically useful DNA-intercalating agents involves the inhibition of the enzyme DNA- topoisomerase I or II. Several detailed SAR studies of acridine-based DNA-intercalating agents suggest that the mode of binding is important and the chromophore intercalate with the DNA base pairs. The chemical modification of acridines such as the introduction of different substitutions or hetero cyclic rings were allowed expansion of research on the structure activity relationship to afford new insight into molecular interactions at the receptor level. ${ }^{12}$ In fact, it is well established that slight structural modification on 9-anilinoacridines may bring various pharmacological effects. Similarly oxazine derivatives also have various biological activities ${ }^{13,14}$ like antimicrobial, anticancer etc. In continuous of our previous research work, ${ }^{15-18}$ on searching new potent cytotoxic agents, we have designed 9-anilinoacridine analogues bearing the oxazine residue on anilino rings for topoisomerase II inhibition by molecular docking studies by using by using Schrodinger suit-2012 Maestro 9.3 version. The results revealed that the newly designed 9-anilinoacridine analogues derivatives exhibited good inhibition with topo II. Generally the topo II inhibitors exhibit anti tumour activity.

A worthwhile approach in this area deals with the use of compounds interacting with DNA and/or inhibiting enzymes critical for cell life and replication. A good example of such a compound is amsacrine a well known antiproliferative agent used to treat some types of cancers including acute adult leukemia.

\section{MATERIALS AND METHODS}

\section{Protein Preparation ${ }^{16}$}

The crystal structure of protein Human Topoisomerase II a (PDB ID: $1 \mathrm{ZXM}$ ) at $1.87 \mathrm{~A}^{\circ}$ was downloaded from the Protein Data Bank (PDB) and was used in order to model the protein structure in this study. In general, the protein structures are refined for their bond orders, formal charges and missing hydrogen atoms, topologies, incomplete and terminal amide groups.

The water molecules beyond $5 \mathrm{~A}^{\circ}$ of the hetero atom were removed. The possible ionization states were generated for the heteroatom present in the protein structure and the most stable state was chosen. The hydrogen bonds were assigned and orientations of the retained water molecules were corrected. Finally, a restrained minimization of the protein structure was carried out using OPLS2005 force field to reorient side-chain hydroxyl groups and alleviate potential steric clashes. The minimization is restrained to the input protein coordinates by a predefined Root Mean Square Deviation (RMSD) tolerance of $0.3 \mathrm{~A}^{\circ}$.

\section{Ligand Preparation}

The structures of the ligands were generated in the CDX format using the tool Chem Draw ultra version 8.0. These ligands were then converted to the mol2 format and subjected to LigPrep module of Maestro in the Schrodinger suite 2013. They were converted from 2D to 3D structures by including stereo chemical, ionization, tautomeric variations, as well as energy minimization and optimized for their geometry, desalted and corrected for their chiralities and missing hydrogen atoms. The bonds orders of these ligands were fixed, and the charged groups were neutralized. The ionization and tautomeric states were generated between $\mathrm{pH}$ of 6.8 to 7.2 using Epik module. In the final stage of LigPrep, compounds were minimized using Optimized Potentials for Liquid Simulations-2005(OPLS-2005) force field in Impact package of Schrodinger until a root mean square deviation of $1.8 \mathrm{~A}^{\circ}$ was achieved. Steepest descent algorithm was used for minimization, followed by conjugate gradient method. A single low energy ring confirmation per ligand was generated and the optimized ligands were used for docking analysis.

\section{Receptorgrid generation}

The ligand ANP (phosphoaminophosphonicacid adenylate ester) was retained in the crystal structure of the prepared protein which was used for the receptor grid construction. The binding box dimensions (within which the centroid of a docked pose is confined) of the protein was set to $14 \mathrm{~A}^{\circ} \times 14 \mathrm{~A}^{\circ} \times 14 \mathrm{~A}^{\circ}$.

\section{Validation of the docking programme}

The accuracy of the docking procedure was determined by finding how closely the lowest energy pose (binding conformation) of the co-crystallized ligand predicted by the object scoring function, Glide score (G Score), resembles an experimental binding mode as determined by X-ray crystallography. Extra precision Glide docking procedure was validated by removing the co-crystallized ligand from the binding site of the protein and redocking the ligand with its binding site. The hydrogen bonding interactions and the root mean square deviation (RMSD) between the predicted conformation and the observed $\mathrm{X}$-ray crystallographic conformation were used for analyzing the results.

\section{Glide Ligand docking}




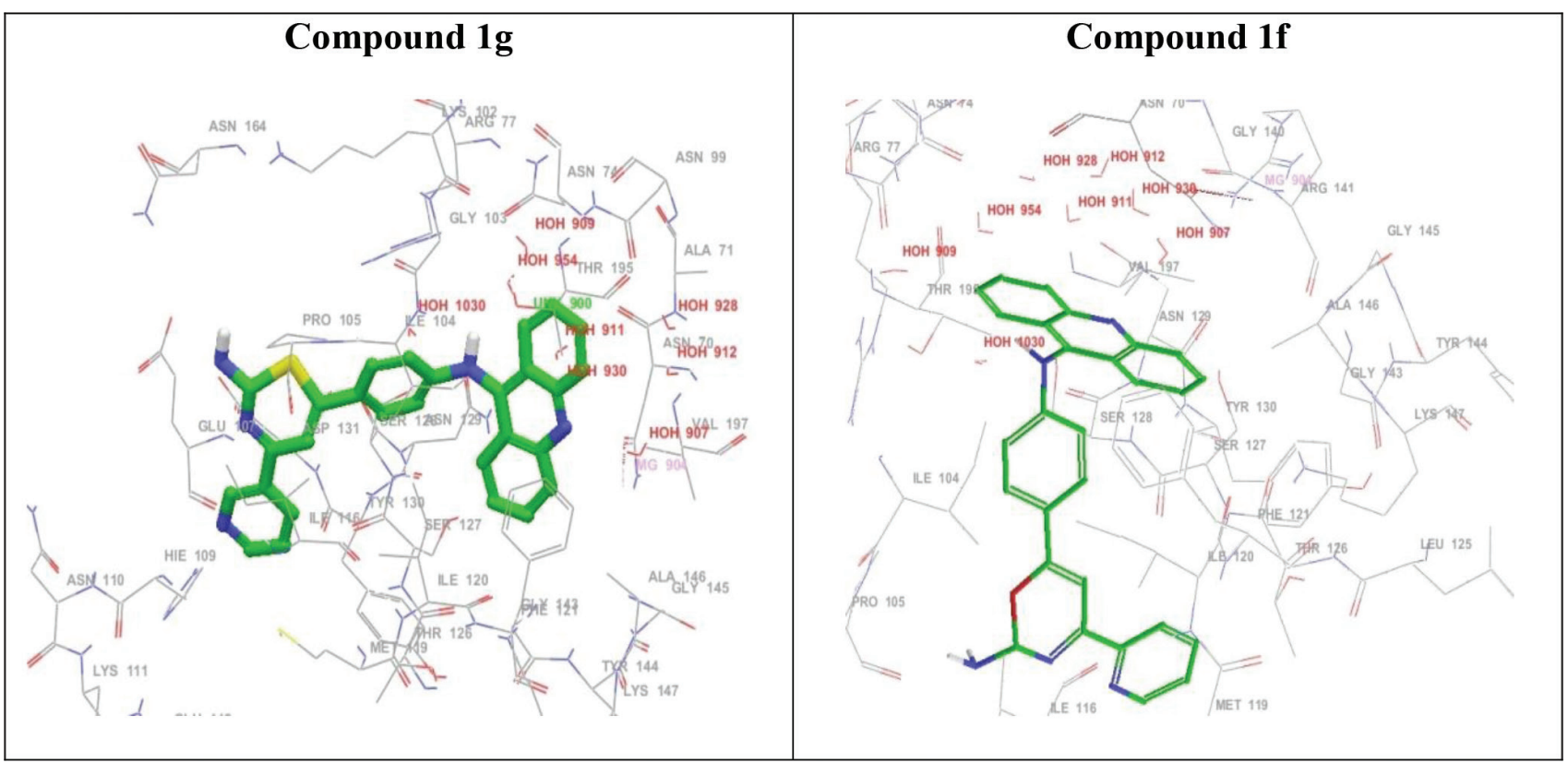

Figure 1: Best affinity mode of docked compounds 1g \& 1f with topoll(1ZXM)

The glide docking of the proposed molecules was carried out using the previously prepared receptor grid and the ligand molecules. The favourable interactions between ligand molecules and the receptor were scored using Glide ligand docking program. All the docking calculations were performed using extra precision (XP) mode and OPLS-2005 force field. The above docking process was run in a flexible docking mode which automatically generates conformations for each input ligand. The ligand poses generated were passed through a series of hierarchal filters that evaluate the ligand's interaction with the receptor. The initial filter test the spatial fit of the ligand to the defined active site, and examines the complementarity of the ligand-receptor interactions using grid-based method patterned after the empirical ChemScore function. This algorithm recognizes favourable hydrophobic, hydrogen-bonding and metal-ligation interactions, and penalizes steric clashes. Poses that pass these initial screens enter the final stage of the algorithm, which involves evaluation and minimization of a grid approximation OPLS non bonded ligand-receptor interaction energy. Finally, the minimized poses were re-scored using Glide Score scoring function.

The XP-Glide score of active compounds were summarized and the fitness scores for each ligand in topo II are compared. When compared with the Glide score of standard compound containing acridine derivative ledacrine which is used as anti cancer agent, as well as potent topoisomerase, most of the proposed compounds have good Glide scores. ${ }^{19}$
The in-silico ADME properties of the proposed compounds were determined by qi prop of Schrodinger software maestro 9.3 version.

\section{RESULTS AND DISCUSSION}

The docking studies of the ligands to protein active sites were performed by an advanced molecular docking program Schrodinger Maestro-9.3 version for determining the binding affinities of the compounds. The designed analogues are docked towards the Topoisomerase-II (1ZXM) in order to ascertain their topoisomerase II inhibition activity. The compounds $1 \mathrm{a}-\mathrm{k}$ (Figure 4) showed good affinity to the receptor when compared with standard acridine derivative ledacrine. The compounds 1c, $1 \mathrm{~g}$ and $1 \mathrm{f}$ have more Glide scores due to more lipophilic character and hydrogen bonding. The compounds 1c, $1 \mathrm{~g}$ and $1 \mathrm{f}$ have more active due to the more lipophilic groups such as dimethoxy phenyl, 2-pyridyl and 3-pyridyl respectively. The results are summarized in the Table 1 . The best affinity modes of the docked compounds (1g, 1f) with Topoisomerase-II having good Glide score are shown in Figure 1. The lipophilic evidence and hydrogen bonding interaction of compound 1c are shown in Figure 2 and 3 respectively. From the Figure 2, the lipophilic evidence was clearly demonstrated. The blue colour region is the more favourable position for the substitution of lipophilic groups to increase the activity and the red colour region is non favourable position for the substitution of lipophilic groups. In the figure 3 the hydrogen bonding is formed between the ligand and the amino acid residues 


\section{Table 1: Docking studies for compounds 1a-k with topoisomerase II (1ZXM)}

\begin{tabular}{|c|c|c|c|c|c|c|c|}
\hline Compound & GScore & $\begin{array}{l}\text { Lipophilic } \\
\text { EvdW }\end{array}$ & HBond & Electro & LowMW & Penalties & RotPenal \\
\hline $1 a$ & -6.35 & -5.14 & -0.94 & -0.27 & 0 & 0 & 0 \\
\hline $1 b$ & -5.7 & -5.36 & 0 & 0.19 & 0 & 0 & 0.15 \\
\hline 1c & -6.79 & -6.04 & -0.7 & -0.27 & 0 & 0 & 0.14 \\
\hline 1d & -6.66 & -5.47 & -0.94 & -0.25 & 0 & 0 & 0 \\
\hline $1 e$ & -6.36 & -5.32 & -0.71 & -0.27 & -0.06 & 0 & 0 \\
\hline $1 f$ & -7.67 & -5.88 & -1.31 & -0.45 & -0.02 & 0 & 0 \\
\hline $1 \mathrm{~g}$ & -8.06 & -5.61 & -1.88 & -0.56 & -0.02 & 0 & 0 \\
\hline $1 \mathrm{~h}$ & -6.16 & -4.89 & -1.03 & -0.21 & -0.02 & 0 & 0 \\
\hline $1 i$ & -6.49 & -5.28 & -0.7 & -0.27 & -0.23 & 0 & 0 \\
\hline $1 j$ & -6.44 & -5.5 & -0.68 & -0.28 & -0.19 & 0 & 0 \\
\hline $1 \mathrm{k}$ & -6.35 & -5.57 & -0.36 & -0.28 & -0.15 & 0 & 0 \\
\hline Ledacrine(std) & -5.24 & -2.94 & -0.22 & -1.66 & -0.42 & 0 & 0 \\
\hline ICRF 193(std) & -6.58 & -1.25 & -0.84 & -3.87 & -0.36 & 0 & 0 \\
\hline Amsacrine(std) & -7.61 & -4.14 & 0 & -5.78 & -0.19 & 2.5 & 0 \\
\hline
\end{tabular}

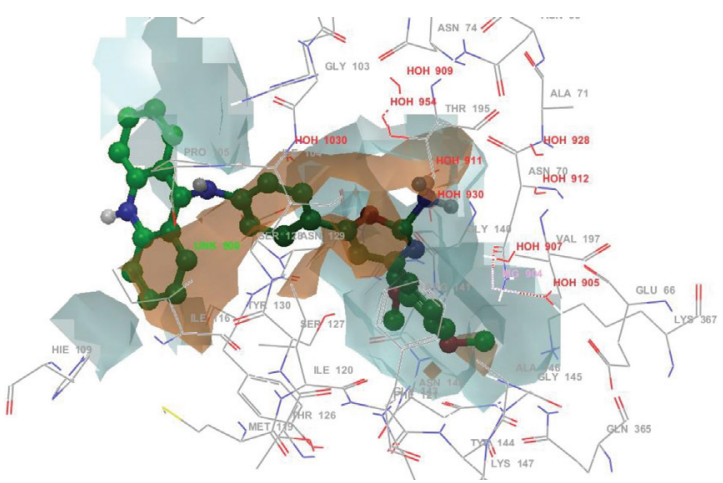

Figure 2: Lipophilic evidence Compound 1c with topoll(1ZXM)

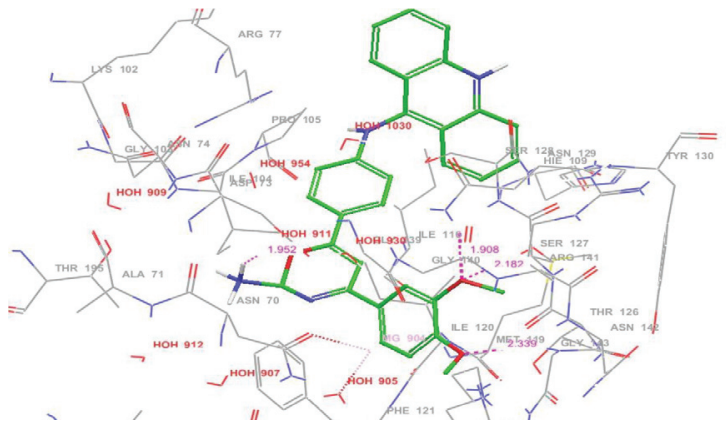

Figure 3: Hydrogen bonding interaction for compound 1c

ASN 70 (H-bond length 1.952), ILE 110 (H-bond length 1.908), GLY 140 (H-bond length 2.182) and MET 119 (H-bond length 2.339).

The ADMET properties for the synthesized compounds can be determined in-silico by using qi prop module of Schrödinger suite 2012. The computed dipole moment of the molecule are in the range of 1.63-5.16. Estimated number of hydrogen bonds that would be donated by
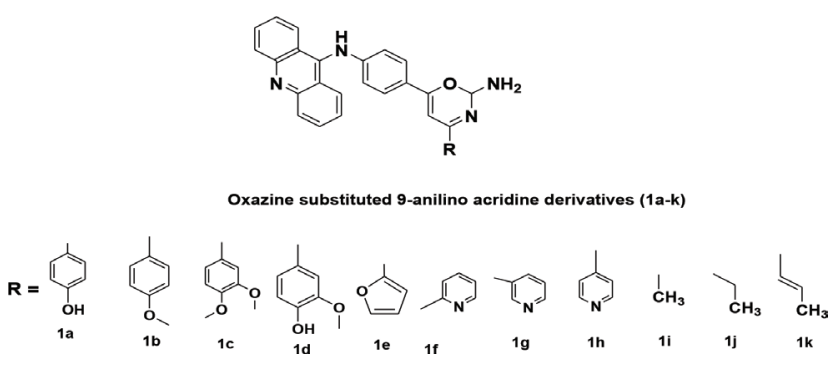

Figure 4: Structure of most active compounds 1a-k

the solute to water molecules in an aqueous solution of the compounds are in the range of 3-4. Estimated number of hydrogen bonds that would be accepted by the solute from water molecules in an aqueous solution of the compounds are in the range of 4.5-6. Predicted octanol/water partition coefficient value of the compounds are in the range of 3.96 -5.6. The compounds has highest QPlogP value. Number of likely metabolic reactions of the compounds are in the range of 3-4. Prediction of binding to human serum albumin for the compounds are in the range of 0.75-1.22. Number of violations of Lipinski's rule of five is $0-2$. Many of the compounds have \% Human Oral Absorption in the range of $79-100 \%$. So almost all the properties of the compounds are within the recommended values. The details of the ADMET properties for the compounds $1 \mathrm{a}-\mathrm{k}$ are shown in the Table 2.

\section{CONCLUSION}

Acridine family includes a wide range of tricyclic molecules with various biological properties. A structure-based pharmacophore model was generated and 
Table 2: insilico ADMET screening for synthesized compounds

\begin{tabular}{|c|c|c|c|c|c|c|c|c|}
\hline Compound & dipole & $\begin{array}{c}\text { Donor } \\
\text { HB }\end{array}$ & $\begin{array}{c}\text { Accpt } \\
\text { HB }\end{array}$ & $\begin{array}{c}\text { logP } \\
\text { o/w }\end{array}$ & $\begin{array}{c}\text { No. of } \\
\text { metabolite }\end{array}$ & $\begin{array}{c}\text { QPlog } \\
\text { Khsa }\end{array}$ & $\begin{array}{c}\text { Rule } \\
\text { of Five }\end{array}$ & $\begin{array}{c}\text { \% Human Oral } \\
\text { Absorption }\end{array}$ \\
\hline 1a & 3.264 & 4 & 5.25 & 4.519 & 3 & 0.925 & 0 & 88.98 \\
\hline 1b & 2.909 & 3 & 5.25 & 5.412 & 3 & 1.179 & 1 & 90.45 \\
\hline 1c & 3.474 & 3 & 6 & 5.607 & 4 & 1.225 & 2 & 78.73 \\
\hline 1d & 2.762 & 4 & 6 & 4.671 & 4 & 0.97 & 0 & 90.49 \\
\hline 1e & 2.43 & 3 & 5 & 4.625 & 3 & 0.942 & 0 & 100 \\
\hline $1 \mathbf{1 f}$ & 1.795 & 3 & 5.5 & 4.773 & 4 & 0.962 & 0 & 100 \\
\hline $\mathbf{1 g}$ & 3.999 & 3 & 6 & 4.429 & 4 & 0.867 & 0 & 93.29 \\
\hline $\mathbf{1 h}$ & 5.164 & 3 & 6 & 4.745 & 4 & 0.975 & 0 & 94.85 \\
\hline $\mathbf{1 i}$ & 2.485 & 3 & 4.5 & 3.962 & 3 & 0.75 & 0 & 93.75 \\
\hline $\mathbf{1 j}$ & 2.346 & 3 & 4.5 & 4.367 & 3 & 0.856 & 0 & 100 \\
\hline 1k & 1.635 & 3 & 4.5 & 4.676 & 3 & 0.971 & 0 & 100 \\
\hline $\begin{array}{c}\text { Recommended } \\
\text { values }\end{array}$ & $1-12.5$ & $0-6$ & $2-20$ & $-2-6.5$ & $1-8$ & $-1.5-1.5$ & max 4 & $>80 \%$ is high \\
\hline
\end{tabular}

Dipole- Computed dipole moment of the molecule,

donorHB - Estimated number of hydrogen bonds that would be donated by the solute to water molecules in an aqueous solution.

accptHB- Estimated number of hydrogen bonds that would be accepted by the solute from water molecules in an aqueous solution

QPlogPo/w - Predicted octanol/water partition coefficient.

\#metab- Number of likely metabolic reactions.

QPlogKhsa- Prediction of binding to human serum albumin.

RuleOfFive Number of violations of Lipinski's rule of five.

$\%$ Human- Oral absorption- Predicted human oral absorption on o to $100 \%$ scale.The prediction is based on a quantitative multiple linear regression model.

validated to obtain active topoisomerase II inhibitors from our self-compiled database of oxazine substituted 9-anilino acridine derivatives.

The docking study revealed that oxazine substituted 9-anilino acridine derivatives showed better alignment at active site by interacting with all crucial amino acid residues. Thus, the in silico method adopted in the present study helped in identifying the lead compounds and also may partly explain their beneficial effect in in vivo study. On this basis, authors recently demonstrated that diverse compounds of the oxazine substituted 9-anilinoacridine series exerted Topoisomerase II inhibitor activity. Results observed in the present study clearly demonstrated that some derivatives of the oxazine substituted 9-anilinoacridine family may exert interesting antitumour activity. The compounds $1 \mathrm{c}, \mathbf{1 f}$ and $\mathbf{1 g}$ have significant antitumour activity with therapeutic potentials and are likely to be useful as drugs after further refinement.

\section{ACKNOWLEDGEMENT}

We thank All India Council for Technical Education, New Delhi for the financial support under Research Promotion Scheme. We also thank our Vice Chancellor Dr.B.Suresh, JSS University, Mysore, Our principal Dr.S.P.Dhanabal, JSS College of pharmacy, Ooty for the technical support.

\section{CONFLICT OF INTEREST}

The authors have no conflict of Interest

\section{REFERENCES}

1. Nadaraj V, Selvi ST, Mohan S. Microwave-induced synthesis and antimicrobial activities of 7,10,11,12-tetrahydrobenzo[c]acridin-8(9H)-one derivatives. Eur J Med Chem. 2009;44:976-80. http://dx.doi.org/10.1016/j. ejmech.2008.07.004 PMid:18718695.

2. Kalirajan R, Muralidharan V, Jubie S, Gowramma B, Gomathy S, Sankar S, Elango K. Synthesis of some novel pyrazole substituted 9-anilino acridine derivatives and evaluation for their antioxidant and cytotoxic activities. J Heterocycle Chem 2012;49:748-54. http://dx.doi.org/10.1002/jhet.848.

3. Rouvier CS, Barret JM, Farrell CM, Sharples D, Hill BT, Barbe J. Synthesis of 9-acridinyl sulfur derivatives: sulfides, sulfoxides and sulfones.Comparison of their activity on tumour cells. Eur J Med Chem. 2004;39:1029-38. http:// dx.doi.org/10.1016/j.ejmech.2004.06.015 PMid:15571864.

4. Rastogi K, Chang JY, Pan WY, Chen $\mathrm{CH}$, Chou TC, et al. Antitumor AHMA linked to DNA minor groove binding agents: synthesis and biological evaluation. J Med Chem. 2002;45(20):4485-93. http://dx.doi.org/10.1021/ jm0200714 PMid:12238927.

5. Bacherikov VA, Chou TC, Dong HJ, Chen CH, Lin YW, Tsai TJ. Potent antitumor $\mathrm{N}$-mustard derivatives of 9-anilinoacridine, synthesis and antitumor evaluation, Bioorg Med Chem Lett. 2004;14:4719-22. http://dx.doi. org/10.1016/j.bmcl.2004.06.080 PMid:15324894.

6. Gamage SA, Tepsiri N, Wilairat P, Wojcik SJ, Figgitt DP, Ralph RK. Synthesis and invitro evaluation of 9-anilino-3,6-diaminoacridines active against a multidrug-resistant strain of the malaria parasite plasmodium falciparum. J Med Chem. 1994;37(10):1486-94. http://dx.doi.org/10.1021/jm00036a014 PMid:8182707.

7. Sondhi SM, Johar M, Nirupama S, Sukla R, Raghubir R, Dastidar SG. Synthesis of sulpha drug acridine derivatives and their evaluation for antianflammatory, analgesic and anticancer acvity. Ind J Chem. 2002;41B:2659-66.

8. Gamage SA, Figgitt DP, Wojcik SJ, Ralph RK, Ransijn A, et al. Structureactivity relationships for the antileishmanial and antitrypanosomal activities of 
1'-substituted 9-anilinoacridines. J Med Chem. 1997;40(16):2634-42. http:// dx.doi.org/10.1021/jm970232h PMid:9258370.

9. Llama EF, Campo CD, Capo M, Anadon M. Synthesis and antinociceptive activity of 9-phenyl-oxy or 9-acyl-oxy derivatives of xanthene, thioxanthene and acridine. Eur J Med Chem. 1989;24:391-96. http://dx.doi. org/10.1016/0223-5234(89)90083-4.

10. Recanatini M, Cavalli A, Belluti F, Piazzi L, Rampa A, Bisi A. SAR of 9-amino1,2,3,4-tetrahydroacridine-based acetyl cholinesterase inhibitors: synthesis, enzyme inhibitory activity, QSAR, and structure-based CoMFA of tacrine analogues. J Med Chem. 2000;43(10):2007-18. http://dx.doi.org/10.1021/ jm990971t PMid:10821713.

11. Goodell JR, Madhok AA, Hiasa H, Ferguson DM. Synthesis and evaluation of acridine- and acridone-based anti-herpes agents with topoisomerase activity. Bioorg Med Chem. 2006;14:5467-80 http://dx.doi.org/10.1016/j. bmc.2006.04.044 PMid:16713270.

12. Harrison RJ, Cuesta J, Chessari G, Read MA, Basra SK, Reszka AP et al. Trisubstituted acridine derivatives as potent and selective telomerase inhibitors. J Med Chem. 2003;46(21):4463-76. http://dx.doi.org/10.1021/ jm0308693 PMid:14521409.

13. Kalirajan R, Sivakumar SU, Jubie S, Gowramma B, Suresh B. Synthesis and biological evaluation of some heterocyclic derivatives of chalcones. Int $\mathrm{J}$ Chem Sci. 2009;1(1): 27-34.

14. Guodong S, Dingben C, Yiliang Z, Manman S, Kai C, Cong J. Synthesis of benzoxazine and 1,3-oxazine derivatives via ligand-free copper(I)-catalyzed

\section{Graphical Abstract}

Novel oxazine substituted 9-anilinoacridines have been designed and docking studies were performed against topoisomerase II. Compounds $\mathbf{1 c}, \mathbf{1 f}, \mathbf{1 g}$ have good Glide score. Insilico ADMET screening also performed.

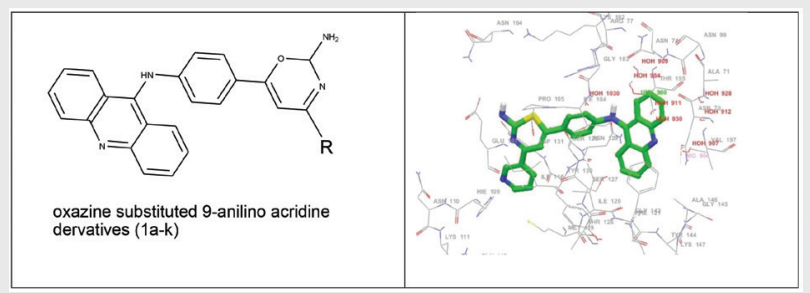

one-pot cascade addition/cyclization reaction. Tetrahedron. 2012;68:166-72. http://dx.doi.org/10.1016/j.tet.2011.10.075

15. Rajagopal K, Muralidharan V, Selvaraj J, Sankar S. Microwave assisted Synthesis, Characterization and Evaluation for their Antimicrobial Activities of Some Novel pyrazole substituted 9-Anilino Acridine Derivatives. Int J Health and Allied Sci. 2013;2(2):81-87. http://dx.doi.org/10.4103/2278344X.115682

16. Kalirajan R, Vivek k, Sankar S, Jubie S. Docking studies, synthesis, characterization of some novel oxazine substituted 9-anilinoacridine derivatives and evaluation for their anti oxidant and anticancer activities as topo isomerase II inhibitors. Eur J Med Chem. 2012;56:217-24. http://dx.doi. org/10.1016/j.ejmech.2012.08.025 PMid:22982526.

17. Kalirajan R, Leela R, Jubie S, Gowramma B, Gomathy S, Sankar S. Microwave assisted synthesis of some novel pyrazole substituted benzimidazoles and evaluation of their biological activities. Indian J Chem. 2011;50B:1794.

18. Kalirajan R, Mohammed rafick MH, Jubie S, Sankar S. Docking studies, synthesis, characterization and evaluation of their antioxidant and cytotoxic activities of some novel isoxazole substituted 9-anilinoacridine derivatives. The Sci World J. 2012;165258, doi:10.1100/2012/165258. http://dx.doi. org/10.1100/2012/165258.

19. Halperin I, Ma B, Wolfson H, Nussinov R. Principles of Docking: An Overview of Search Algorithms and a Guide to Scoring Functions. PROTEINS: St Fun Gen. 2002;47:409-43 http://dx.doi.org/10.1002/prot.10115.abs http://dx.doi. org/10.1002/prot.10115 PMid:12001221.

\section{SUMMARY}

- Oxazine substituted 9-anilinoacridines are designed for Topo II inhibitors.

- These compounds were docked with 3D structure of Topoisomerase II (PBD ID: 1ZXM) retrieved from Protein Data Bank.

- The docking study results revealed that the above compounds showed better alignment at active site by interacting with all crucial amino acid residues.

\section{About Authors}

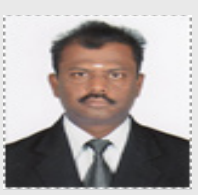

Dr. R.Kalirajan: Is currently Asst. professor at JSS College of Pharmacy, Ooty. He has experience in the areas of computer aided drug design and Synthetic Medicinal chemistry.

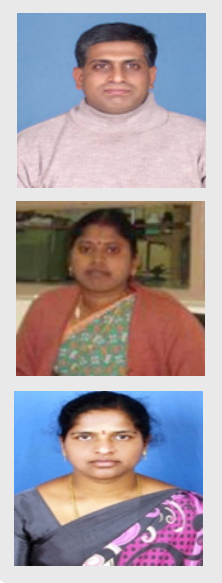

Dr. S. Sankar: Is currently professor at JSS College of Pharmacy, Ooty. He has experience in the areas of Natural products isolation and Synthetic chemistry.

Dr. S. Jubie: Is currently Asst. professor at JSS College of Pharmacy, Ooty. He has experience in the areas of computer aided drug design and Synthetic Medicinal chemistry.

Dr. B. Gowramma: Is currently Asst. professor at JSS College of Pharmacy, Ooty. He has experience in the areas of Analytical methods and Synthetic chemistry.

Cite this article: Kalirajan R, Sankar S, Jubie S, Gowramma B. Molecular Docking studies and in-silico ADMET Screening of Some novel Oxazine substituted 9-Anilinoacridines as Topoisomerase II Inhibitors. Indian J of Pharmaceutical Education and Research. 2017;51(1):110-115. 